\title{
LA COMUNICACIÓN DURANTE LA INTERVENCIÓN DIDÁCTICA DEL ENTRENADOR. CONSIDERACIONES PARA EL DESARROLLO DEL CONOCIMIENTO TÁCTICO Y LA MEJORA EN LA TOMA DE DECISIONES EN BALONCESTO
}

\author{
Communication during the didactic intervention of the coach \\ considerations for the development of tactial knowledge \\ and the improvement in decision making in basketball \\ Damián Iglesias ${ }^{1}$, David Cárdenas ${ }^{2}$ y Francisco Alarcón ${ }^{3}$ \\ 1 Facultad de Formación del Profesorado. Universidad de Extremadura \\ 2 Facultad de Ciencias de la Actividad Física y del Deporte. Universidad de Granada \\ 3 Facultad de Ciencias de la Actividad Física y del Deporte. UCAM \\ DIRECCIÓN PARA CORRESPONDENCIA \\ David Cárdenas \\ Facultad de Ciencias de la Actividad Física y del Deporte. Universidad de Granada \\ Ctra. de Alfacar, s/n. 18011 Granada \\ dcardena@ugr.es
}

Fecha de recepción: Junio 2007 • Fecha de aceptación: Noviembre 2007

\begin{abstract}
RESUMEN
El rendimiento deportivo en baloncesto depende de múltiples factores, de diferente naturaleza: motriz, física, psicológica. Tras muchos años en los que la principal preocupación de los técnicos fue el desarrollo de las destrezas motoras, la producción científica reciente ha demostrado la importancia de los procesos cognitivos en la toma de decisiones, la necesidad de fomentar estrategias orientadas a proporcionar autonomía al jugador o la influencia decisiva de las emociones en la elección adecuada de las respuestas. Bajo este prisma se ha producido un cambio sustancial en el proceso de entrenamiento que contempla los procesos cognitivos asociados a las habilidades perceptivas y tácticas como un factor fundamental vinculado al rendimiento. En el presente artículo se aborda el rol que debe desempeñar el entrenador durante su intervención didáctica, con un especial interés en los procesos de comunicación, como medio para conducir al deportista en la construcción del conocimiento, el descubrimiento de las soluciones a los problemas de juego y, por tanto, el desarrollo de los mecanismos cognitivos necesarios para ello.
\end{abstract}

Palabras clave: Baloncesto, entrenamiento, conocimiento táctico, toma de decisiones, procesos de comunicación.

\section{ABSTRACT}

The sport performance in basketball depends on multiple factors, of different nature: motor, physical, psychological. After many years in which the main preoccupation of the coaches was the development of the motor skills, the recent scientific production has demonstrated the importance of the cognitive processes in the decision making, the necessity to foment oriented strategies to provide autonomy to the player or the decisive influence of the emotions in the right election of the motor answers. Under this prism a substantial change in the training process has taken place that contemplates to the cognitive processes associate to the perceptive and tactical abilities like a tie fundamental factor to the performance. The present article treats on the role that the coach must carry out during its didactic intervention, with a special interest in the processes of communication, like average leading to the sportsman in the construction of the knowledge, the discovery of the solutions to the game problems and, therefore, the development of necessary the cognitive mechanisms for it.

Key words: Basketball, training, tactical knowledge, decision making, processes of communication. 


\section{Introducción}

El baloncesto, como deporte de equipo donde tienen lugar habilidades abiertas, presenta una serie de particularidades especificas diferentes a otros deportes que debemos tener en cuenta al analizar cómo obtener rendimiento $y_{\text {, por }}$ tanto, cómo formar y entrenar. El jugador desarrolla su actuación deportiva en un entorno complejo, caracterizado por la presencia de multitud de estímulos a los que debe atender de forma preferente, concretamente cinco defensores, cuatro compañeros y un móvil que se desplaza por el terreno de juego por acción de los participantes. Además, con la dificultad añadida de que estos nueve jugadores presentes en la pista modifican su posición continua y simultáneamente en el tiempo. Esta caracteristica del juego demanda a los jugadores una elevada actividad perceptiva y decisional, para poder actuar adecuadamente. De aquí se deriva la necesidad de plantear un entrenamiento por un lado conceptual y por otro perceptivo, que permita al jugador conocer cuáles son los aspectos relevantes del juego en los que tiene que focalizar su atención en cada momento, puesto que las limitaciones de su campo visual no le van a permitir acceder a toda la información disponible en el entorno de juego y, menos aún, poder dotar de significado a todas esas unidades o porciones informativas.

De este modo, no podemos atribuir el rendimiento únicamente al desarrollo de las destrezas motoras. Los procesos cognitivos asociados a las habilidades perceptivas y tácticas configuran un factor importante vinculado al rendimiento, que hemos de contemplar en el entrenamiento.

Pero, ¿qué significa jugar bien a baloncesto desde el punto de vista del rendimiento individual? Podemos entender que "jugar bien consiste en elegir la opción de juego más adecuada en cada momento y ser capaz de llevarla a cabo de forma eficiente y consistente a lo largo de un encuentro" (Gréhaigne, Goodbout y Bouthier, 2001). Esta capacidad táctica para elegir la acción pertinente en cada situación requiere un análisis previo del entorno de juego, de alta dificultad, por parte del jugador. Y, por otro lado, ser ca- paz de llevar a cabo la opción de juego elegida requiere el dominio de las destrezas técnicas específicas que permitan materializar lo que el jugador, desde un punto de vista cognitivo, se ha planteado ejecutar. Por tanto, el desarrollo de la pericia en baloncesto incluye la capacidad de tomar decisiones apropiadas y el dominio de determinadas destrezas técnicas (Thomas, 1994).

También, es preciso señalar que la complejidad del desarrollo de estos procesos de análisis, elección de la opción más adecuada y realización técnica, se agravan por la necesidad de realizarse en situaciones de déficit de tiempo, dado que las situaciones de juego se alternan rápidamente y lo que en un principio parecia que podia ser una buena acción, instantes después supondría un grave error.

Por tanto, el jugador de baloncesto debe estar continuamente tomando decisiones, fundamentalmente, con relación a dos cuestiones:

"OUÉ HACER": Selección de la respuesta en juego.

"CÓMO HACERLO": Ejecución de la acción técnica más conveniente.

La psicología cognitiva establece que el proceso de toma de decisiones por parte de un sujeto está mediatizado por las estructuras de conocimiento almacenadas en la memoria. De tal forma que los sujetos, a partir de su formación y acumulación de experiencia a través de la práctica deliberada, van mejorando su conocimiento tanto conceptual como procedimental sobre el juego, es decir, su capacidad para optar por la acción de juego más propicia en cada situación. Los sujetos van desarrollando su pericia por medio de la práctica, elaborando procedimientos de selección cada vez más acertados y de forma más rápida (Thomas, Gallagher y Thomas, 2001).

Finalmente, hay que matizar que el proceso por el cual el sujeto es capaz de tomar decisiones en el deporte depende del tiempo disponible para la procesar la información. Hay situaciones en las que el jugador, pese a que el tiempo disponible, como hemos indicado, es escaso, cuenta con el suficiente para percibir y decidir de forma consciente, por lo que el proceso podemos entenderlo como "reflexivo". En otros casos el tiempo es tan reducido que el sujeto se ve obligado a decidir la respuesta utilizando mecanismos cognitivos reactivos en los que no es posible analizar la situación y las consecuencias de las posibles respuestas, de forma consciente. Esto justificaria que muchos jugadores acertaran en su respuesta, pero al ser preguntados por los factores que les ayudaron a resolver la situación no sepan contestar. ¿Significa esto que el sujeto acertó aleatoriamente? Podría darse el caso de que así fuera, pero ¿qué sucede si el deportista acierta en condiciones similares con frecuencia y sigue sin saber responder a la pregunta? ¿Quiere esto decir que por el hecho de que su forma de actuar sea inconsciente (no es capaz de explicar verbalmente las claves por las que actuó de esa manera) no hubo intervención de su inteligencia motriz?

En la actualidad los estudios en el campo de las neurociencias han demostrado que el ser humano está dotado de mecanismos filogenéticos que le ayudan a procesar la información tanto de forma consciente (cuando hay tiempo) como inconsciente (cuando el tiempo es mínimo), que originalmente servian como medios para garantizar la supervivencia. Por este motivo, cuando un individuo conduce un coche puede estar concentrado en los problemas que acontecieron durante la jornada laboral o las cosas que le quedan por hacer y, súbitamente, esquivar un perro que se cruza en la carretera. Por un lado, su capacidad atencional múltiple le permite atender a varias cosas al mismo tiempo $y_{\text {, por otro, }}$ cuando la situación demanda reaccionar de manera inmediata, su cerebro procesa la información de forma inconsciente pero eficaz (aunque no siempre, pues dependerá de la pericia al volante, fruto, entre otras cosas, de la experiencia acumulada).

Al primero de los casos le vamos a llamar, para entendernos, inteligencia reflexiva; al segundo, inteligencia intuitiva. Por fortuna en nuestro baloncesto hace algún tiempo que podemos disfrutar al ver jugadores inteligentes e intuitivos (aquellos capaces de anticiparse a los oponentes aunque no sepan explicar cómo lo hacen).

Llegados a este punto, cabe plantearse algunas cuestiones: ¿Qué puede hacer el entrenador para ayudar a que sus jugado- 
res adquieran el conocimiento táctico que les ayudará a jugar inteligentemente cuando haya tiempo para analizar las situaciones de juego? ¿Cómo se puede mejorar la toma de decisiones más intuitivas de los jugadores? El objetivo de este trabajo es analizar la figura del entrenador bajo un enfoque didáctico, desarrollando pautas prácticas de comportamiento que pueden ayudar a mejorar la formación táctica del jugador.

\section{Los modelos de}

entrenamiento deportivo

Enseñar a los jugadores a tomar decisiones correctas en el juego es una tarea de gran complejidad. Han sido diferentes los intentos por desarrollar programas de entrenamiento que combinen el aprendizaje de las destrezas técnicas con los aspectos de toma de decisiones en el juego para, de este modo, poder formar sujetos competentes.

Tradicionalmente, el entrenamiento ha tenido un enfoque orientado hacia el desarrollo de las destrezas técnicas, relegando a un papel secundario la comprensión de la dinámica y lógica interna del juego. Este modelo técnico se componía, fundamentalmente, de tres fases sucesivas: actividad introductoria, fase de desarrollo de la destreza, y juego. La comprensión del juego no sólo es necesaria, sino imprescindible para rendir en baloncesto; el juego exige comprender cuándo y dónde utilizar la técnica, además de dominarla. Sin embargo, también debemos tener en cuenta que al menos un cierto nivel de dominio de las destrezas motoras es necesario para llevar a cabo comportamientos tácticos y estratégicos en el juego (Turner y Martinek, 1999).

Frente al modelo técnico apareció el denominado modelo comprensivo. Bajo este nuevo modelo se defiende la necesidad de que tanto la comprensión del juego y el desarrollo del conocimiento táctico o procedimental precedan al desarrollo de las destrezas técnicas, es decir, las ideas relacionadas con "qué hacer" deberían ir antes que las de "cómo hacerlo". Esta aproximación establece que el desarrollo de las destrezas técnicas no ha de ser uno de los fines primeros, sino que pone énfasis en el papel del conocimiento y la comprensión. Bajo este modelo, el entrenamiento comienza con "juegos modificados" y se introducen reglas generales de comportamiento con la intención de desarrollar un conocimiento táctico del juego y la capacidad de tomar decisiones en cuanto a "qué hacer". Por tanto, se pone el énfasis en el aprendizaje práctico, auténtico y con significado.

Nuestra propuesta se sitúa más cerca de esta segunda línea de actuación, al aplicar los principios del constructivismo (Cárdenas y Pintor, 2001), por un lado, y los modelos de procesamiento de la información, por otro. A continuación, trataremos de dar respuesta a las preguntas que planteábamos inicialmente. Para ello, pensamos que el entrenador debe tener en cuenta dos aspectos fundamentales: el diseño de las tareas que propone en los entrenamientos y su intervención didáctica. Aunque resulta evidente su relación, puesto que el diseño de una actividad debe recoger cuál debe ser la actuación del entrenador durante su puesta en práctica y, de igual modo, hacer referencia a la intervención docente supone una afinidad directa con las características de la tarea

Figura 1. Factores que influyen en el diseño de las tareas de enseñanza-aprendizaje en baloncesto.

\section{FACTORES RELACIONADOS CON EL JUGADOR}

LOS QUE DETERMINAN EL NIVEL DE PARTIDA EN EL PROCESO DE APRENDIZAJE

- Conocimientos previos.

- Experiencias acumuladas.

- Nivel de juego (capacidad técnico-táctica).

- Características físicas.

- Características psicológicas.

LOS QUE DETERMINAN LA PREDISPOSICIÓN DEL SUJETO

- Motivación.

- Autoestima

- Nivel de activación

- Capacidad de concentración.

LOS QUE DETERMINAN LAS POSIBILIDADES DEL ALUMNO

- Capacidad perceptiva.

- Capacidad cognitiva-actividad mental del alumno.

- Capacidad para la elaboración motriz de respuestas.

\section{FACTORES RELACIONADOS CON EL ENTRENADOR}

LOS QUE DETERMINAN LAS METAS A CONSEGUIR

- Planteamiento de objetivos.

- Selección de contenidos.

LOS RELACIONADOS CON EL PROCEDIMIENTO DE ENSEÑANZA

- Principios metodológicos.

- Pautas de intervención didáctica. 


\section{FACTORES RELACIONADOS CON EL CONTENIDO}

LA DIMENSIÓN COGNITIVA Y EL NIVEL DE DIFICULTAD DE LA TAREA

- Demandas perceptivas.

- Demandas relacionadas con la capacidad de procesamiento.

- Demandas relacionadas con la ejecución motriz.

LOS ASPECTOS ORGANIZATIVOS DE LA TAREA

- Organización del espacio.

- Organización del tiempo.

- Organización del grupo (tipo de participación y rotaciones).

- Organización del material.
Tal como se apuntaba anteriormente, necesitamos desarrollar dos tipos de mecanismos cognitivos: los que permiten tomar decisiones razonadas, pues existe tiempo suficiente (aunque siempre es reducido en baloncesto) para tomar la decisión, y los que hacen posible la toma de decisiones más intuitivas, sin que intervenga la consciencia.

Tanto para unos como otros las consignas respecto al diseño de la tarea van a ser similares. Lo que debe cambiar es la intervención del entrenador; en unos casos se les ayudará a reflexionar sobre lo que hacen y lo que deberian hacer $y$, en otros, se espera que el sujeto explore y busque respuestas de manera libre.

\section{Criterios de calidad}

Como referencias generales podemos afirmar que las tareas de enseñanzaaprendizaje deberían cumplir los siguientes criterios de calidad:

1. Deben estar adaptadas a las características de los jugadores a los que van dirigidas.

2. Deben promover la consecución de los objetivos propuestos.

3. Deben garantizar un índice de participación elevado.

4. Deben integrar el mayor número de contenidos de aprendizaje posibles.

5. Deben asegurar una implicación cognitiva del jugador durante la práctica.

6. Deben permitir la libre exploración y la resolución personal de las situaciones de juego que se generen.

7. Deben garantizar la transferencia de los aprendizajes.

8. Deben aprovechar al máximo los recursos humanos y materiales existentes (Goñi, 2007).

\section{La intervención didáctica del entrenador}

Resulta de gran interés, y así lo han demostrado estudios de investigación centrados en analizar la conducta docente del entrenador, que éste planifique su intervención docente en la pista y, posteriormente, reflexione sobre ella de cara a futuras actuaciones. Bajo este proceso continuado de trabajo, el entrenador mejorará la eficacia en su labor de entrenamiento, consiguiendo aumentar, en consecuencia, el rendimiento de sus jugadores.

Se han establecido diversas clasificaciones sobre cuáles han de ser estas competencias docentes que tiene que desarrollar el entrenador y mejorar a través de mecanismos reflexivos, que, sin duda, están relacionadas con la eficacia en el proceso de entrenamiento, además de la propias tareas en sí mismas. Las competencias más significativas se pueden agrupar, fundamentalmente, en torno a cuatro ideas (basado en Delgado, 1990 y Piéron, 1999):

- La información que el entrenador trasmite al inicio de la sesión de entrenamiento y al modificar una misma situación de juego bajo nuevas consignas, 0 iniciar una nueva (información inicial).

- La información que el entrenador suministra durante el desarrollo de las tareas propuestas (feedback).

- La rentabilidad del tiempo durante el proceso del entrenamiento (tiempo de práctica).

- La actuación eficaz desde el punto de vista organizativo, incluyendo materiales y agrupaciones de jugadores (eficacia organizativa).

- Promover un clima de relaciones positivas entre el entrenador y los jugadores, y de los jugadores entre sí (clima social).

A partir de estos presupuestos, se han desarrollado protocolos, más o menos sistematizados, que ayudan y guian al entrenador durante el proceso de entrenamiento. Bajo esta idea, y acorde con los planteamientos constructivistas del aprendizaje, Cárdenas (1999) sugirió una secuencia concreta de intervención didáctica estructurada en ocho fases y adaptada de Pozo (1996), autor que procede del ámbito educativo): planteamiento de la situación, libre exploración, reflexión inducida, provo-
Figura 2. Fases del proceso de intervención didáctica. Tomado de Cárdenas (1999). Adaptado de Pozo (1996).

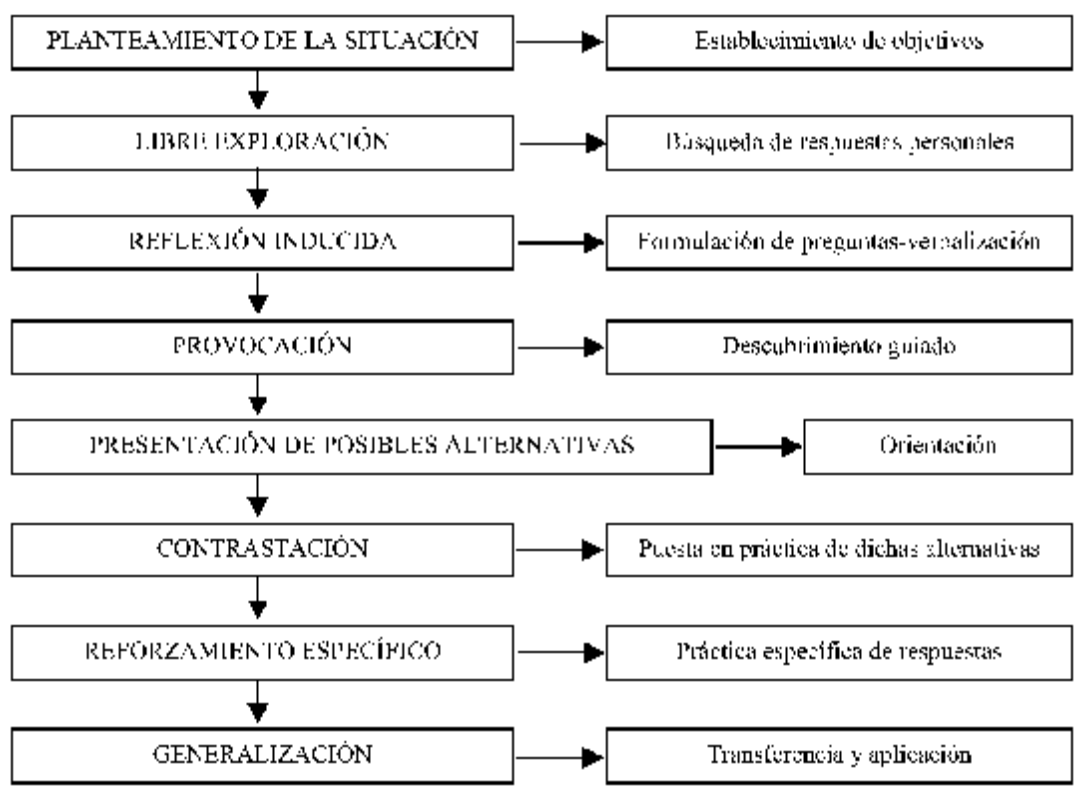


cación, presentación de posibles alternativas, contraste, refuerzo especifico y generalización (figura 2).

Como nuestro objetivo es analizar cómo puede contribuir el entrenador a la adquisición del conocimiento táctico y a la mejora en la toma de decisiones de sus jugadores durante los entrenamientos, a través de sus competencias docentes, centraremos nuestra atención, en primer lugar, sobre las destrezas de comunicación verbal: información inicial y feedback.

\section{Información inicial}

La información que se aporta antes de producirse la tarea, o información inicial, tiene como objetivo su presentación (Delgado, 1991; Pierón, 1999; Sánchez, 1992). Existe una problemática importante sobre la idea de cómo se debe informar al jugador durante el proceso de entrenamiento para favorecer su actuación competente en el juego.

Probablemente, algunos elementos de aprendizaje se puedan aprender por el hecho de practicar, sin necesidad de recibir instrucción directa (Ruiz, 1997) y, para otros, será necesario desarrollar una conducta verbal, más o menos prevista de antemano, por parte del entrenador. En este sentido, cualquier entrenador comprenderá que la mera práctica de situaciones concretas de juego permitirá el desarrollo de la habilidad para manejar el balón sin dar información precisa de cómo hacerlo, pero difícilmente favorecerá la adquisición del dominio técnico necesario para hacer un lanzamiento eficaz a media o larga distancia.

Piéron (1999) extrae que se puede dividir la presentación de tareas según el aspecto que comunique en:

- Comunicar el objetivo. El entrenador informa sobre el "para qué" de las actividades de aprendizaje.

- Comunicar el objeto (contenido). En este caso la información va dirigida a lo que van a realizar los jugadores. La información sobre el contenido puede tener un carácter conceptual (para qué sirve un bloqueo directo), procedimental (para hacer un BD debo hacer...) o actitudinal (debes respetar al compañero). Además la información sobre el contenido puede hacer más hincapié en la fase perceptiva (cuando juegues un $\mathrm{Bl}$, fijate en cómo te defiende tu oponente), en la toma de decisiones (si el defensor te persigue, recordad qué debéis hacer) o en la propia ejecución (para conseguir que bloqueen a tu oponente debes pasar el bloqueo hombro con hombro).

- Comunicar la técnica. Con esta categoría se refiere a las operaciones y las formas de realizar la tarea. Sobre las operaciones el entrenador puede dar información sobre cómo organizar el material, el espacio, el tiempo, y los jugadores. A esto Fernández (2002) lo llama dar información sobre el escenario o el ambiente donde se va a desarrollar el reto. Esta información que da el entrenador sobre el entorno tiene el objetivo de informar al jugador sobre cómo se organizarán las condiciones del entorno para realizar la tarea motriz.

Autores como Blázquez (1982) realizan una clasificación de la información inicial según una mayor o menor definición de los elementos básicos que constituyen una tarea. Estos elementos son los objetivos que se pretende conseguir, las operaciones que se deben realizar y el acondicionamiento del medio y material. Así la información inicial del entrenador puede que defina al máximo estos elementos, por lo que no queda ninguna duda de lo que hay que realizar, siendo los jugadores meros reproductores de lo que se les solicita.

Este tipo de intervención del entrenador responde a un modelo instructivo y persiguen el aprendizaje de patrones motores estereotipados (Díaz, 1999). El entrenador puede especificar en su información inicial alguno de los elementos que las constituyen, dando orientaciones a los jugadores. Esta intervención es más propia de técnicas de enseñanza como la resolución de problemas.

Por último, el profesor puede no especificar ninguno de los tres elementos, o lo realiza de manera escasa, con lo que el grado de intervención del entrenador es mínimo, dejando la mayoría de las decisiones a los jugadores. Estas tareas fomentan la creatividad del jugador (Díaz, 1999).

\section{Feedback}

Una vez que los jugadores están realizando las tareas propuestas, el entrenador puede intervenir suministrando información durante el transcurso de la práctica a través del aporte de feedback. En función de las características de esta información y de la intención con la que se aporta, podemos diferenciar varias tipologías de feedback (Sanz, 2003):

Específico. Definido como aquel que se dirige de forma concreta y directa hacia algún elemento de aprendizaje. Podemos diferenciar:

- Descriptivo. Supone la aportación de información que contiene detalles sobre la ejecución del movimiento realizado. Por ejemplo: "estabas desequilibrado cuando has lanzado".

- Explicativo. A través de él, se aporta información sobre las causas del error en la ejecución. Por ejemplo: "tu problema es que al botar no te proteges con el cuerpo".

- Evaluativo. Aporta una estimación cualitativa de la actuación acompañada de una justificación. Por ejemplo: "el lanzamiento ha sido oportuno, porque el defensor estaba lejos, y la canasta, cerca".

- Prescriptivo. Aporta información sobre cómo se deben realizar las próximas ejecuciones. Por ejemplo: "itienes que cambiar de defensor tras bloqueo directo!".

- Interrogativo. Supone realizar una pregunta al jugador sobre la ejecución que ha realizado, para intentar hacerle reflexionar y que tome consciencia sobre su propia ejecución. “'Por dónde pasó el bloqueo directo tu oponente directo?".

Inespecífico. Referido a una información de carácter afectivo y relacionado con la dimensión emocional del jugador. Por ejemplo: "ivamos!", "ibien!"

Si nuestra intención es tratar de conectar con la formación táctica, las explicaciones que realiza el entrenador, tanto al inicio de cada actividad, como durante o al finalizar la misma, deben tener un carácter de aplicación práctica, y ésta variará dependiendo del momento en el que nos encontremos dentro de la progresión de enseñanza propuesta por Cárdenas (1999). 
En la fase de planteamiento de la situación, cuando todavía el jugador no ha iniciado su actividad motriz, el entrenador ya puede empezar a favorecer su comprensión sobre el juego. Sugerimos que esta información previa a la tarea, sobre todo en etapas de formación, sea de carácter general, vinculada a planteamientos exploratorios, donde cada jugador, efectivamente, tenga muy claro cuál es el objetivo que se persigue, pero sin limitar su proceso de búsqueda abierta. Es importante que durante la información inicial no se especifique cuáles son las operaciones necesarias para conseguir el objetivo, pues esto va a generar en el jugador la necesidad de una búsqueda de soluciones diferentes. Es lo que Blázquez (1982) Ilama tareas semidefinidas de tipo II. Probablemente se comprenda mejor con un ejemplo. Si el objetivo de una tarea es la mejora de las arrancadas del jugador con balón en situaciones desde parado, se puede plantear a los jugadores una situación de $1 \times 1$, en la que se les informe inicialmente de este objetivo matizándoles la necesidad de dificultar la acción de su defensor para conseguir una ventaja inicial, pero evitando aportar información sobre cómo hacerlo, es decir, que deben realizar acciones de engaño previas, o utilizando para ello los pivotes, etc.

La fase de reflexión inducida se podria definir como un feedback de la fase anterior, puesto que sólo es verbal, y el objetivo es hacer reflexionar al jugador sobre la actuación que ha tenido en la práctica libre. Esta reflexión va encaminada a activar en el jugador los conocimientos previos que posee sobre el objetivo que se quiere enseñar. Siguiendo el ejemplo de la tarea anterior, se le preguntará al jugador sobre cómo ha solucionado los problemas para conseguir superar a su defensor.

En la información inicial que se da en la fase de provocación, el entrenador, al igual que en la fase anterior, no debe especificar las operaciones necesarias para conseguir el objetivo de la tarea. La diferencia estriba en que, en esta ocasión, en la información sobre cómo condicionar el entorno, ésta debe ir encaminada a provocar en el jugador la necesidad de buscar las posibles vías para conseguir el objetivo. Para ello se puede dar información sobre una norma de actuación. Por ejem- plo para la tarea anterior se puede definir la siguiente norma: "prohibido que el balón durante la acción de bote contacte en el suelo más de una vez". Esta limitación reglamentaria conduce al jugador a pensar en sus recursos disponibles para generar espacio libre desde parado, evitando la indeseable conducta de botar inmediatamente que se recibe el balón, sin analizar previamente la situación de juego. Por otro lado, le obliga a utilizar las acciones de pivote como medio para arrancar desde parado o fintar dicha intención generando espacio libre para el tiro, si el defensor reacciona. Como podemos ver, la norma impuesta favorece la implicación cognitiva del sujeto en la tarea, el desarrollo de su capacidad táctica individual, la comprensión de algunos principios específicos del juego individual (botar sólo cuando sea necesario) y el aprendizaje de acciones especificas, como los pivotes, arrancadas y fintas. Se trata pues, de que la información aportada por el entrenador que precede al desarrollo de la actividad estimule cognitivamente al jugador y le obligue a pensar antes de coger el balón. No obstante y dado que, como indicamos anteriormente, también es necesario desarrollar los mecanismos cognitivos más intuitivos, hay que decir que la tarea propuesta favorecerá la aparición de situaciones que obliguen al jugador a tomar decisiones en un tiempo tan reducido y, por tanto, el desarrollo de este tipo de mecanismos. En cuanto al tipo de feedback que es más recomendable ofrecer en esta fase, éste debería seguir siendo interrogativo, pero con la intención de focalizar la atención del jugador en los elementos más importantes para conseguir el objetivo, del tipo: "¿cómo está orientado el defensor?, ¿qué pie tiene más adelantado?, ¿cómo está el defensor, separado o pegado a ti?

En la fase de presentación de nuevas alternativas, además de especificar el objetivo a conseguir y las condiciones del entorno, en la información inicial, el entrenador debe presentar las operaciones necesarias para lograr dicho objetivo, aunque para ello no tenga que especificarlas directamente, sino que, mediante preguntas, éste guie al jugador hacia ellas. Esto se realizará tanto en la información inicial, como durante la realiza- ción o al finalizar la tarea. En conexión con el ejemplo anterior, el entrenador puede aprovechar un pequeño periodo de tiempo para preguntar al jugador acerca de "qué" hacer para conseguir espacio libre para el tiro exterior, o para penetrar de forma efectiva con las limitaciones impuestas, etc. En definitiva, el entrenador promueve que el jugador reflexione sobre lo que hace e interiorice lo que practica para aprender.

Tanto en la fase de contrastación, como en la fase de generalización, la información inicial es similar a la de libre exploración, donde las condiciones del entorno no se modifican en relación con las establecidas por el reglamento de cada deporte. La diferencia entre una y otra estriba en que, en la fase de generalización, la información sobre las condiciones del entorno va encaminada a generar situaciones y contextos de juego diferentes a los planteados hasta ese momento para buscar las máxima transferencia (Cárdenas y Pintor, 2001). El feedback utilizado en estas fases será inespecífico (Sanz, 2003), e irá encaminado a reforzar positivamente la elecciones adecuadas de los jugadores, mediante expresiones de carácter afectivo (jeso es!, ibien hecho!).

Por último, en la fase de reforzamiento específico, las tareas que se utilizan son definidas (Blázquez, 1982), es decir, la información inicial va dirigida a especificar todos los elementos de la tarea, dirigiendo la actuación de los jugadores de manera clara hacia la respuesta más correcta, que eso sí, previamente ha sido analizada y reflexionada por los jugadores. Un ejemplo de esta información inicial podría ser: "jugamos 1x1 interior donde el defensor debe orientarse siempre ofreciendo el fondo al atacante y debe de caer en la primera finta que le realice el atacante; el atacante debe intentar superar al oponente hacia el centro, pero con una finta previa hacia el fondo". El feedback en esta fase tiene como objetivo tanto explicar como prescribir lo que el jugador debe hacer, como por ejemplo: "utiliza el pivote cruzado cuando el defensor esté cerca, para proteger el balón).

En resumen, la comunicación entrenador-jugadores tendrá siempre como objetivo informar sobre qué hacer, pero con un carácter abierto y favoreciendo la indaga- 
ción desde el punto de vista individual. Es decir, la información suministrada por el entrenador debe exigir al jugador una implicación cognitiva relacionada con el "qué hacer". Esta demanda nunca deberá ser relegada por parte del entrenador, para propiciar aprendizajes que tengan significado $y$, así, posteriormente, construir otros conceptos nuevos más complejos. Para ello es necesario plantear situaciones donde haya un primer momento de toma de contacto y exploración para, posteriormente, tras detener la actividad, buscar comportamientos razonados en función de lo que ha ocurrido en el juego, a través de mecanismos que provoquen la reflexión del jugador. Más que corregir los errores que observa de forma directa, propiciará que sea el jugador quien analice sus respuestas en la actividad, tome consciencia del error y busque alternativas. Será fundamentalmente a través del feedback de tipo interrogativo como consigamos que el jugador realice un acto de autoanálisis y reflexión sobre sus actuaciones en el juego, que facilite el desarroIlo tanto del conocimiento conceptual como procedimental. Preguntando al jugador ¿por qué?, le conduciremos hacia un comportamiento razonado, consciente e intencionado en el juego. Aprender a reconocer momentos clave para lanzar a canasta, pasar o botar en situaciones de juego reducidas, deberá ser previo a la comprensión de que algunos planteamientos de táctica colectiva en el juego de $5 \times 5$ tienen como finalidad provocar algunas de estas situaciones.

En ocasiones, como hemos visto, a pesar de utilizar este planteamiento activo de búsqueda por parte del jugador, es posible que éste no alcance a conocer la respuesta a la situación-problema que le plantea la actividad. En estos casos, se utilizaría la fase de reforzamiento especifico, donde otros tipos de feedback de carácter descriptivo, explicativo o evaluativo, deben ser el recurso a emplear. Es decir, realizar un proceso de búsqueda más convergente, pero siempre favoreciendo el aprendizaje constructivo y con significado. Aunque el feedback prescriptivo puede resultar de interés para determinadas situaciones o jugadores en concreto, debe ser un recurso a utilizar de forma esporádica, cuando no es posible acceder a soluciones de otro modo, porque este tipo de feedback suplanta la implicación cognitiva del jugador; es el entrenador quien dice lo que hay que hacer.

Por otra parte, no debemos olvidar la importancia del feedback inespecífico en el proceso de entrenamiento. A pesar de no ir dirigido de forma directa hacia aspectos relacionados con el juego, supone un impulso y una ayuda psicológica muy importante.

A veces, se trata más de ayudar a comprender que se puede mejorar, de favorecer la autoestima del jugador, de generar confianza..., que de realmente intervenir de forma más técnica.

Además este tipo de feedback es importantísimo para la mejora de las decisiones intuitivas, puesto que el aprendizaje de estas conductas se produce mediante técnicas de reforzamiento positivo, donde el jugador, de manera inconsciente, asocia una determinada respuesta (la correcta) con una sensación de éxito gracias al reforzamiento afectivo utilizado por el entrenador.

En relación con lo anterior, es importante reparar sobre algunas cuestiones cuando hacemos referencia a la comunicación del entrenador (Rodríguez, 2000):

- La comunicación no es un proceso unidireccional, es decir, no sólo consiste en transmitir, sino también en escuchar.

- La comunicación está comprendida tanto por mensajes verbales como por los no verbales.

- La comunicación se compone de dos dimensiones, contenido y emoción, la primera, relacionada con el hecho sustantivo del mensaje, y la segunda, respecto a la sensación del jugador al recibirlo. De hecho, en la actualidad, los avances neurocientíficos (Damasio, 2006) nos están obligando a replantearnos la necesidad de concederle una mayor importancia al desarrollo de las habilidades psicológicas que permiten el control emocional, debido a las evidencias empíricas que demuestran la influencia de las emociones en la toma de decisiones del ser humano. Probablemente en un futuro no muy lejano pasaremos a construir los procesos de aprendizaje no desde planteamiento de objetivos motrices sino emocionales 0 , en cualquier caso, de forma paralela.
Torre (2002) nos ofrece una taxonomía que ayuda a reflexionar sobre estos aspectos, estableciendo que durante la comunicación verbal hemos de tomar en consideración tanto las calidades de la voz, es decir, el volumen, la duración..., como los sonidos y ruidos que podemos emitir (suspiros, risas...). Es evidente que una palabra o un conjunto de palabras pueden tener una interpretación distinta en función de estas caracteristicas, relativas a lo que se denomina comunicación paraverbal.

También resulta de mucho interés ser conscientes de los mensajes que transmitimos de forma no verbal. Este tipo de comunicación, menos tenida en cuenta y casi olvidada, puede ser mucho más influyente que la verbal. El lenguaje del cuerpo o la comunicación corporal se estructura, de forma general, en los gestos, los movimientos, las posturas y el contacto corporal (Torre, 2002). En ocasiones, el entrenador puede emitir mensajes contradictorios con la voz y el cuerpo. Es necesario buscar una coherencia entre ambos tipos de comunicación, y ser capaz de hacer un uso adecuado de ambos, por separado, aunque en la mayoría de los casos la comunicación no verbal se emplea para apoyar y reforzar el contenido del mensaje emitido de forma verbal.

Por último, haremos referencia al uso y estructuración del espacio como elemento de comunicación. Por ejemplo, una distribución adecuada, que permita y facilite el contacto visual entre todo el equipo, en situaciones de información inicial, favorecerá la dinámica interna del grupo. Para el caso del feedback, la situación de acercamiento al jugador, incluso de contacto físico con él, propiciará una sensación de cercanía, de confianza, de agradabilidad..., muy importante en la formación del jugador.

\section{Futuras líneas de investigación}

A partir de la propuesta de intervención didáctica para el entrenador sugerida en este manuscrito, que se ha centrado de manera especifica en el diseño de las tareas de entrenamiento $y_{\text {, especial- }}$ mente, en las destrezas de comunicación (información inicial y feedback), enunciamos a continuación algunas posibles 
vías de investigación con el objetivo de poder contrastar empíricamente estas reflexiones.

1. Diseñar estudios de carácter longitudinal en los que se intervenga a partir de tareas de entrenamiento con diferentes características. De este modo, los datos aportados contribuirán a mejorar nuestro conocimiento sobre qué tipo de actividades y con qué estructura se propicia de un mejor modo la formación táctica de los sujetos.

2. Plantear investigaciones, también de corte experimental, en las que se apliquen tratamientos con diferentes instrucciones verbales, tanto durante la información inicial como en el feedback. Así, podremos seguir avanzando en la construcción de una estructura de comunicación verbal destinada a facilitar la adquisición del conocimiento procedimental.

\section{BIBLIOGRAFÍA}

Cárdenas (1999). Los factores relacionados con el profesor-entrenador en un enfoque constructivista para la enseñanza del baloncesto en la etapa escolar. Espacio y Tiempo, 27, 16-21.

Cárdenas, D. y Pintor, D. (2001). La iniciación al baloncesto en el medio escolar. En Ruiz, F, García, A. y Casimiro, A.J. (Eds.). La iniciación deportiva basada en los deportes colectivos. Nuevas tendencias metodológicas. Madrid, Gymnos.

Damasio, A. (2006, 3a edición). El error de Descartes. Barcelona. Crítica.

Delgado, M.A. (1990). Influencia de un entrenamiento docente durante las prácticas docentes sobre alguna de las competencias del profesor de Educación Física. Tesis doctoral. Universidad de Granada.

Gréhaigne, J.F., Godbout, P., y Bouthier, D. (1999). The foundations of tactics and strategy in team sports. Journal of Teaching in Physical Education, 18, 159-174.

Piéron, M. (1999). Para una enseñanza eficaz de las actividades fisico-deportivas. Barcelona. Inde.

Pozo, I. (1996). Aprendices y maestros. La nueva cultura del aprendizaje. Madrid: Alianza Editorial.
Rodríguez, M. (2000). La comunicación en la enseñanza del tenis. Salamanca. Tesitex.

Ruiz, L.M. (1997). Deporte y aprendizaje. Procesos de adquisición y desarrollo de habilidades. Madrid, Aprendizaje Visor.

Sanz, D. (2003). Análisis y optimización de la conducta del entrenador de tenis en silla de ruedas de alta competición durante el proceso de entrenamiento. Tesis doctoral. Universidad de Extremadura.

Thomas, K.T. (1994). The development of sport expertise: From Leeds to MVP legend. Quest, 46, 211-222.

Thomas, K.T., Gallagher, J.D., y Thomas, J.R. (2001). Motor development and skill acquisition during childhood and adolescence. En Singer, R. N., Hausenblas, H.A. y Janelle, C.M. (Eds.) Handbook of Sport Psychology (Second Edition). John Wiley \& Sons, INC.

Torre, E. (2002). La comunicación educativa. Retos, 3, 37-43.

Turner, A. y Martinek, T.J. (1999). An investigation into teaching games for understanding: Effects on skill, knowledge, and game play. Research Quarterly for Exercise and Sport, 70, 286-296. 